\title{
An update from the 2019 ESMO meeting
}

\author{
Andreas Pircher
}

The 2019 annual congress of the European Society for Medical Oncology (ESMO) took place in Barcelona, Spain from 27 September to 1 October under the tagline "Translating science into better cancer patient care". Thereby, the major aims of the congress were to translate, as soon as possible, the advances in clinical science into real actions for the best care of patients as well as to highlight the importance of a multidisciplinary treatment approach in modern oncology.

The personal highlights for lung cancer treatment were summarized by Horvath et al. [1], concluding that ESMO 2019 fuelled the lung cancer community with inspiring new data contributing to a more individualized treatment approach. This includes fine-tuning of immunotherapy combinations or biomarker-stratified immunotherapy use in the first-line setting for patients without driver mutations. In genetically driven non-small cell lung cancer (NSCLC), the major highlight was the presentation of the long-awaited overall survival data of the FLAURA study, which were however, controversially discussed. New data were also presented on rare subgroups of lung cancer patients with ROS or NTRK alterations, which have emerged as important drivers and further strengthen genetic testing.

The ESMO 2019 highlights in bladder and renal cancer summarized by Niedersüß-Beke highlight the impact of the plethora of combination therapies [2]. In addition, new concepts as combination therapies with antibody drug conjugates are evolving in

\footnotetext{
A. Pircher, MD PhD $(\bowtie)$

Internal Medicine V, Department of Hematology and Oncology, Medical University of Innsbruck, Anichstr. 35, 6020 Innsbruck, Austria

andreas.pircher@i-med.ac.at
}

early clinical studies and further validation is eagerly awaited as first response data are highly encouraging.

In line with the other tumor types also in gastrointestinal oncology genetically defined subgroups including BRAF V600E-mutated colorectal cancer (CRC) and fibroblast growth factor (FGF)/receptor (FGFR)altered cholangiocellular carcinomas have shown encouraging results when treated with targeted agents as summarized by Amann et al. [3]. In addition, in hepatocellular carcinoma combination therapies will soon become part of clinical reality.

In breast cancer, two important studies investigating the combination of the CDK4/6 inhibitors abemaciclib or ribociclib in combination with fulvestrant reported prolonged overall survival over endocrine therapy alone in a mixed population of first- and second-line patients with metastatic hormone-receptor positive breast cancer. In highly aggressive triplenegative breast cancer (TNBC) in the neo-adjuvant setting, pembrolizumab significantly improved pathological complete response rates regardless of PDL1 status when used in combination with standard chemotherapy compared to chemotherapy alone. Bartsch et al. [4] summarize that this trial could possibly refine our daily practice in neoadjuvant TNBC treatment.

To summarize, ESMO 2019 again proved to be an important meeting in Europe for clinicians, researchers and the pharmaceutical industry from all over the world in order to share information, to learn about the latest advances in cancer as well as to translate recent scientific novelties into better patient care. Detailed highlights are discussed in the four articles included in this ESMO 2019 highlights editorial. Enjoy reading about the most recent advances and see you at the 2020 meeting in Madrid. 


\section{editorial}

Conflict of interest A. Pircher has received speaker's fee and honoraria for advisory boards from Astra Zeneca, BMS, Roche, Pfizer, Takeda and MSD.

\section{References}

1. Horvath L, Pircher A.ESMO2019-personalnon-small lung cancer highlights. memo. 2020. https://doi.org/10.1007/ s12254-020-00589-x.

2. Niedersüß-Beke D. My personal highlights of ESMO 2019 regarding urogenital cancer. memo. 2020. https://doi.org/ 10.1007/s12254-020-00605-0.

3. Amann A. ESMO 2019-personal highlights in gastrointestinal cancer. memo. 2020. https://doi.org/10.1007/ s12254-020-00603-2.
4. Bartsch R. ESMO 2019: breast cancer. memo. 2020. https:// doi.org/10.1007/s12254-020-00588-y.

Publisher's Note Springer Nature remains neutral with regard to jurisdictional claims in published maps and institutional affiliations.

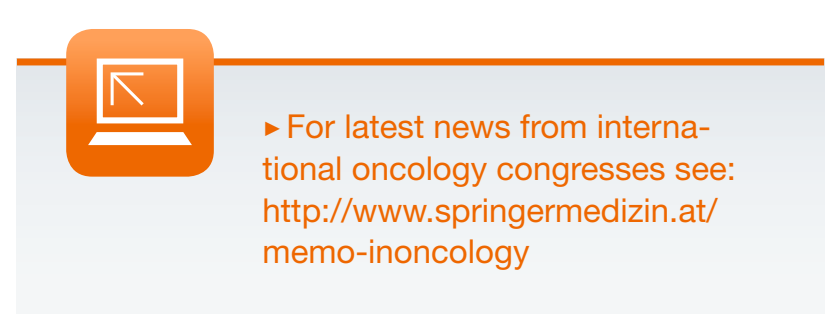

